Check for updates

Cite this: RSC Adv., 2018, 8, 15687

Received 5th March 2018 Accepted 12th April 2018

DOI: $10.1039 / c 8 r a 01913 d$

rsc.li/rsc-advances

\section{Preparation and characterization of emamectin benzoate nanoformulations based on colloidal delivery systems and use in controlling Plutella xylostella (L.) (Lepidoptera: Plutellidae)}

\author{
Ali Shoaib, ${ }^{\text {ab }}$ Muhammad Waqas, ${ }^{a}$ Asem Elabasy, ${ }^{\text {ab }}$ Xinlai Cheng, ${ }^{a}$ Qianqian Zhang ${ }^{a}$ \\ and Zuhua Shi (D)*a
}

Colloidal delivery systems have been widely used as carriers for controlled delivery of pesticides to improve the efficacy and photostability of natural and semi-synthetic pesticides. In this study, we have synthesized emamectin benzoate nanoformulations ( $E B+N F s$ ) depending on polymeric nanocapsules (PNC) and two types of the nanosilica, mesoporous nanosilica (MCM-48) and silicon dioxide nanoparticles (SNPs) as carriers for the emamectin benzoate (EB). The fabricated nanoformulations were characterized by using $X$-ray diffraction analysis, Fourier transform infrared spectroscopy, particle size, zeta potential, morphology, absolute recovery (AR), entrapment efficiency (EE), UV stability and release kinetics. The obtained results showed that the carriers had a remarkable loading ability for EB and improved the EB photostability. The EE\% of nanoformulations were $92.84 \%, 87.45 \%$ and $71.19 \%$ for emamectin benzoate polymeric nanocapsules (EB + PNC), emamectin benzoate SNPs (EB + SNPs) and emamectin benzoate MCM-48 (EB + MCM-48) respectively. The insecticidal activity of EB + NFs against Plutella xylostella showed that the EB + SNPs was more effective than other EB + NFs and EB alone. The $L_{50}$ values were $0.18,4.03,8.49$ and $11.06 \mathrm{mg} \mathrm{L}^{-1}$ for $E B+S N P s, E B+M C M-48, E B+P N C$ and $E B$ respectively. The obtained results suggest the colloidal delivery systems that used in this study could improve the efficacy and photostability for EB, and they are able to overcome the disadvantage of the natural and semisynthetic pesticides such as environmental sensitivity and to increase the efficacy of pesticides, which eventually leads to reduce the dosage of pesticides needed, reducing the number of applications required in comparison to conventional formulations.

\section{Introduction}

Herbivorous insects are very destructive pests for the important crops and their production. These insects cause damage by feeding on seedlings, germinating seeds and flowers. The chemical pesticides are useful for protecting the crops from insect damage during the growing season. However, indiscriminate use of them has led to several environmental problems, including serious health hazards to humans and animals, development of insecticide resistance, destruction of beneficial insects and accumulation of pesticide residues in different environmental compartments.

In the recent years, natural and semi-synthetic pesticides have gained interest as a promising alternative to conventional pesticides for pest insect control, ${ }^{1}$ but these pesticides exist

${ }^{a}$ Key Laboratory of Molecular Biology of Crop Pathogens and Insects, Ministry of Agriculture, Institute of Insect Sciences, Zhejiang University, 866 Yuhangtang Road, Hangzhou 310058, China. E-mail: zhshi@zju.edu.cn

${ }^{b}$ Department of Pesticides, Plant Protection Research Institute, Agricultural Research Centre, Cairo, Egypt obviously some disadvantages, including low activity and short persistent under various environmental conditions such as sunlight, humidity and rainfall. Emamectin benzoate (EB) is a semi-synthetic derivative of abamectin of the avermectin family of 16-membered macrocylic lactones. This epi-methyl amino derivative showed increased effectiveness against a broad spectrum of lepidopterous and coleopterous pests with application rates in active ingredient (a.i.) ranging between 8.4$16.8 \mathrm{~g} \mathrm{ha}^{-1} \cdot{ }^{2,3}$ Unfortunately, the avermectin compounds are degraded rapidly from the environment after application. The binding compounds to the soil are rapidly decomposed by the soil microorganisms after fast photolysis on plant surfaces. Additionally, the current commercial formulations of EB are sensitive to the light and temperature. These problems limit the use of EB in agriculture sector because an insecticide should persist in the field for enough time to ensure adequate control of pests. ${ }^{2}$ Therefore, it is an urgent need to produce formulations that could meet the requirements of high efficiency and prolonged protection. 
Colloidal delivery systems such as polymeric nanocapsules (PNC) and polymeric nanospheres (PNS) have been used to overcome these disadvantages and to improve the insecticidal properties according to the principle of controlled release formulations (CRFs). CRFs have the ability to reduce the environmental hazards of excessive use of pesticides around the world to protect crops. In many cases the use of CRFs could reduce the total applied amount of pesticide a.i. by reducing the concentration and time of application leading to reduce economic cost as compared with conventional formulations. Consequently, it could lower its residue on agricultural products and the risks to humans and the environment. ${ }^{4-6}$

Colloidal delivery systems show high efficiency as a means of efficiently delivering one or a mixture of active ingredients to their site of action. Furthermore, PNC can reduce the side effects of the insecticides and improve the photostability of active ingredients. ${ }^{7}$ A lot of work has been done on colloidal delivery systems in agricultural sector. Acephate polymeric nanocapsule synthesized with polyethylene glycol (PEG-400) showed increasing solubility in water, increasing stability and efficiency at a lower dose, reducing the economic cost for each application, and decreasing acephate toxicity to beneficial insects when compared with the commercial acephate formulation. ${ }^{8,9}$ Polyethylene glycol (PEG) coated nanoparticles (NPs) loaded with garlic essential oil were efficient against adult Tribolism castaneum..$^{10}$ The control efficiency against adult $T$. castaneum was remained over $80 \%$ after five months with NPs loaded garlic essential oil but was only $11 \%$ with garlic essential oil alone. The EB microcapsules based on a copolymer matrix of silica-epichlorohydrin-carboxymethylcellulose could protect effectively EB against photo- and thermal degradation and thereby increase their efficacy against Myzus persicae. ${ }^{\mathbf{1 1}}$

The mesoporous silica nanoparticulates (MSNs) can increase the stability, dispersity, and the controlled release of pesticide compounds under environmental condition. The photostability of avermectin was improved by the porous hollow silica nanoparticle (PHSNs) carriers entrapping it into the hollow core of the nanoparticle carriers. ${ }^{12}$ Also the PHSNs improved the efficacy of controllable release, photo-stability and water solubility of abamectin by modifying the porous structure of silica nanoparticles, which is useful to enhance the bioavailability and decrease the residues of pesticides. ${ }^{13}$ In another study, it was found that the CRFs based on MSNs were successfully used to adsorb and release imidacloprid. ${ }^{\mathbf{1 4}}$ Also, polymeric encapsulation based Functional Nano-Dispensers (FNDs) of imidacloprid showed that the FNDs were an effective releasing materials for insecticides as compared with a standard commercial formulation, providing an acceptable level of protection for at least 10 days. Additionally, the FNDs could reduce the amount of imidacloprid required to cause similar mortality of Diaphorina citri as compared with the commercial formulation. Moreover, FNDs have greater potential as a cost-effective solution against a number of pests. ${ }^{15}$

In this work, three novel functionalized EB + NFs emamectin benzoate polymeric nanocapsules (EB + PNC), emamectin benzoate silicon dioxide nanoparticles (EB + SNPs) and emamectin benzoate mesoporous nanosilica (EB + MCM-48), were prepared based on colloidal delivery systems to improve the EB stability under various environmental conditions such as sunlight and humidity. The EB + NFs were characterized with regard to their particle size distribution, zeta potential (ZP), entrapment efficiency (EE), and morphology. Photostability studies were also performed for all EB + NFs in vitro condition. The toxicity of three EB + NFs against diamondback moth, Plutella xylostella L. (Lepidoptera: Plutellidae), were evaluated and compared with EB alone.

\section{Experimental details}

\section{Materials}

Emamectin benzoate (70\%) was kindly provided by Institute of Pesticide \& Environmental Toxicology, Zhejiang University. Ethyl cellulose, sodium silicate $\left(\mathrm{Na}_{2} \mathrm{SiO}_{3}\right)$, sulfuric acid $\left(\mathrm{H}_{2} \mathrm{SO}_{4}\right)$, cetyltrimethylammonium bromide (CTAB), tetraethyl orthosilicate (TEOS, 98\%) and ammonium hydroxide $\left(\mathrm{NH}_{4} \mathrm{OH}\right)$ solution were purchased from Sangon Biotech (Shanghai) Co., Ltd, (Shanghai, China). All organic solvents (methanol, ethanol, acetonitrile, acetic acid, and dichloromethane) [highperformance liquid chromatography (HPLC) grade], Pluronic ${ }^{\circledR}$ F-127 (nonionic surfactant), sorbitan monostearate (Span 60) and sorbitan monooleate (Tween 80) were purchased from Sigma-Aldrich (St. Louis, MO, USA). Ultrapure water was produced in our laboratory using a Milli-Q System (18 M $\Omega$ ) (Millipore Corp., Bedford, MA, USA).

\section{Preparation of EB + NFs}

Preparation of EB + PNC. The EB + PNC was prepared according to Forim's method with some modifications. ${ }^{1}$ Nanoemulsion was prepared by vigorous homogenization. The mixture of $0.2 \mathrm{~g}$ a.i. of EB dissolved in $5 \mathrm{~mL}$ methanol and $0.2 \mathrm{~g}$ of Span 60 poured in $100 \mathrm{~mL}$ water was stirred through UltraTurrax homogenizer (IKA T 10 B S25 basic Ultra-Turrax; IkaWerke, Germany) at $28000 \mathrm{~g}$ for $5 \mathrm{~min}$ to produce EB nanodrops. After a brief period of stabilization, the solution of $0.5 \mathrm{~g}$ of ethyl cellulose dissolved in $20 \mathrm{~mL}$ ethanol was poured into nanodrops of EB under magnetic stirring by mini air compressor AS18BK with airbrush HS-30K (Haosheng Pneumatic Machinery Co., Ltd, Ningbo, China), under pressure 30 psi. After stirring above mixture for $10 \mathrm{~min}$, the third solution of $0.2 \mathrm{~g}$ of Tween $80 \mathrm{in} 20 \mathrm{~mL}$ water was poured to the previously made solution under magnetic stirring for $10 \mathrm{~min}$.

Preparation of EB + SNPs. The SNPs was prepared using solgel technique in accordance with the Music's method with some modifications. ${ }^{16}$ A total of $0.2 \mathrm{~g}$ equivalent sodium silicate $\left(\mathrm{Na}_{2} \mathrm{SiO}_{3}\right)$ was diluted in $300 \mathrm{~mL}$ ultrapure water, and $0.2 \mathrm{~g}$ equivalent sulfuric acid $\left(\mathrm{H}_{2} \mathrm{SO}_{4}\right)$ was diluted in $200 \mathrm{~mL}$ ultrapure water. The $\mathrm{H}_{2} \mathrm{SO}_{4}$ solution was added drop by drop into the sodium silicate solution. The mixture was mixed through a magnetic stirrer for $45 \mathrm{~min}$ to form a nanosilica gel. The gel was washed six times in a filter paper with ultrapure water to remove excessive sodium sulfate $\left(\mathrm{Na}_{2} \mathrm{SO}_{4}\right)$ from the mixture under vacuum filtration. The gel was dried by using an ALPHA 1-2 LD plus freeze dry (Martin Christ Gefriertrocknungsanlagen 
GmbH, Osterode am Harz, Germany) for $24 \mathrm{~h}$ to obtain SNPs powder.

The SNPs loaded with EB were prepared by a freeze drying technique, colloidal solution from SNPs and EB (a.i.) were mixed at a ratio of $1: 1$ by weight. Firstly, $0.8 \mathrm{~g}$ SNPs was diluted in $200 \mathrm{~mL}$ ultrapure water, and the SNPs colloidal solution was sonicated for $30 \mathrm{~min}$ to ensure the nanoparticles are separated from each other. Then, $0.8 \mathrm{~g}$ EB (a.i.) was dissolved in $20 \mathrm{~mL}$ methanol. The EB solution was added drop by drop into the colloidal SNPs under continuously stirred condition by the magnetic stirrer at speed $600 \mathrm{rpm}$ for $2 \mathrm{~h}$ at room temperature. After $2 \mathrm{~h}$ the EB could be completely lodged on the surface of SNPs and then the mixture was dried by using the ALPHA 1-2 LD plus freeze dry for $24 \mathrm{~h}$ to get EB + SNPs powder.

Preparation of EB + MCM-48. The MCM-48 nanoparticles were prepared using the method of Kim et al. ${ }^{17}$ Briefly, $0.5 \mathrm{~g}$ CTAB and $2.05 \mathrm{~g}$ Pluronic F127 were dissolved in a solution of ultrapure water $(96 \mathrm{~mL})$, ethanol $(34 \mathrm{~mL})$ and $29 \%$ (by weight) ammonium hydroxide solution $(10.05 \mathrm{~mL})$ at room temperature. After complete dissolution, $1.8 \mathrm{~g}$ of TEOS was added into the mixture at once. After $1 \mathrm{~min}$ of mechanical stirring at $1000 \mathrm{rpm}$, the mixture was kept at a static condition for $24 \mathrm{~h}$ to generate silica nanoparticles. Resulting white precipitates were collected, centrifuged and washed twice with ethanol, and dried determined by High Performance Liquid Chromatograph (HPLC), Agilent 1260, equipped with UV detector at $245 \mathrm{~nm}$ and HPLC column (Zorbax Eclipse XDBC18 $(150 \times 4.6 \mathrm{~mm}$ i.d., $5 \mu \mathrm{m}$ particle size, stainless steel)). The mobile phase consists of acetonitrile (99.8\%): acetic acid (0.1\%) (80:20 v/v). The injection volume was $20 \mu \mathrm{L}$ with a flow rate of $1.0 \mathrm{~mL} \mathrm{~min}^{-1}$, the oven temperature maintained at $30{ }^{\circ} \mathrm{C}$.

The percentage of entrapment efficiency (EE\%) of EB in EB + PNC was determined by measuring the concentration of the free unloaded compound in the aqueous phase of the EB + PNC. Centrifugation was carried out using a tube filter containing $0.22 \mu \mathrm{m}$ pore cellulose acetate membrane (Costar Spin-X, Corning Inc.). A total of $0.5 \mathrm{~mL}$ of colloidal EB + PNC suspension was placed in the outer chamber of the filter assembly, and the assembly was then centrifuged at $2700 \mathrm{~g}$ for $15 \mathrm{~min}$ at $15{ }^{\circ} \mathrm{C}$. The encapsulated compounds were remained in the outer chamber, whereas the aqueous medium containing the free unloaded EB was moved to the sample recovery chamber through the filter membrane. After separation, $0.2 \mathrm{~mL}$ of the aqueous medium was dried. The dried product was dissolved in $2 \mathrm{~mL}$ of methanol, and subsequent concentration was determined by HPLC as described earlier by Forim et al. ${ }^{1}$ The EE\% was subsequently calculated using the following equation:

$$
\mathrm{EE} \%=\frac{\text { total quantity of emamectin benzoate }- \text { quantity of free of emamectin benzoate in the aqueous medium }}{\text { total quantity of emamectin benzoate }} \times 100
$$

under vacuum. Finally the dried precipitates were calcined at $550{ }^{\circ} \mathrm{C}$ for $5 \mathrm{~h}$.

Loading experiments were carried out in methanol as EB is highly soluble in methanol. EB (a.i.) and the MCM-48 carriers were mixed at a weight ratio of $2: 1$ (a.i. EB : MCM-48). The MCM-48 (0.5 g) were suspended in $100 \mathrm{~mL}$ methanol and sonicated for $30 \mathrm{~min}$. EB (1 g a.i.) was dissolved in $50 \mathrm{~mL}$ methanol, then the EB solution was added drop by drop into MCM-48 suspensions. Whole solution was continuously stirred by magnetic stirrer at $600 \mathrm{rpm}$ for $30 \mathrm{~min}$ at room temperature. The mixed solution was shaken for $24 \mathrm{~h}$, a time period found to be sufficient to reach equilibrium. After $24 \mathrm{~h}$ impregnation, the suspensions were used to evaluate EB + MCM-48. ${ }^{14}$

\section{Determination of emamectin benzoate content}

Determination of emamectin benzoate in EB + PNC. The total amount percentage of absolute recovery (AR\%) of $\mathrm{EB}$ in $\mathrm{EB}$ + PNC was determined using the following method. First, $0.1 \mathrm{~mL}$ of the $\mathrm{EB}+\mathrm{PNC}$ was dissolved in $0.9 \mathrm{~mL}$ of ethanol for two hours. After polymer dissolution, the solution was centrifuged (centrifuge $5417 \mathrm{R}$; Eppendorf, Germany) at $20800 \mathrm{~g}$ for $30 \mathrm{~min}$ at $20{ }^{\circ} \mathrm{C}$. After phase separation, $0.5 \mathrm{~mL}$ of the supernatant was dried under vacuum (concentrator plus, Eppendorf, Germany). Then, the dried compound containing EB was dissolved in $2 \mathrm{~mL}$ of methanol, and the total amount of EB was
Determination of EB in EB + SNPs and EB + MCM-48. The total amount percentages of absolute recovery (AR\%) of $\mathrm{EB}$ in the nanoformulations were determined using the following method. First, $1 \mathrm{~mL}$ of the EB + SNPs or EB + MCM-48 solution was dissolved in $10 \mathrm{~mL}$ of dichloromethane, and the samples were sonicated for $30 \mathrm{~min}$ and then the mixture was magnetically stirred at $1000 \mathrm{rpm}$ for $3 \mathrm{~h}$ to ensure complete extraction of the EB from samples. After this, $1 \mathrm{~mL}$ from the samples were centrifuged (centrifuge 5417 R; Eppendorf, Germany) at $20800 \mathrm{~g}$ for $30 \mathrm{~min}$ at room temperature. After phase separation, $0.1 \mathrm{~mL}$ of the supernatant was dried under vacuum (concentrator plus, Eppendorf, Germany). Then, the dried compound containing EB was dissolved in $1 \mathrm{~mL}$ methanol, and the total amount of EB was determined by HPLC as done in EB + PNC.

The percentage of entrapment efficiency (EE\%) of EB was determined by measuring the concentration of the free unloaded compound in the aqueous phase of the colloidal solution according to Forim et al. as previously mentioned method. ${ }^{1}$

\section{Characterization of EB + NFs}

The surface morphology was observed using scanning electron microscope (SEM, TM-1000, Hitachi, Japan) and transmission electron microscope (Tecnai ${ }^{\mathrm{TM}}$ Spirit TEM, FEI Company, Hillsboro, OR, USA). X-ray diffraction (XRD) measurements were performed using a multipurpose X-ray diffractometer 
(XRD, X'Pert PRO, PANalytical, Almelo, The Netherlands) with $\mathrm{Cu}$ radiation $(\lambda=1.54 \mathrm{~A})$ at $40 \mathrm{kV}$ and $40 \mathrm{~mA}$. The samples were scanned from 1 to $90^{\circ} \theta$, and the scanning rate was $2^{\circ} \theta$ per min with step size of $0.02^{\circ} \theta$. Fourier transform infrared spectrophotometer (FT-IR) (Vector 22, Bruker, Germany) was used to identify the different functional groups presented in the samples. Particle size and zeta potential values were evaluated by NanoPlus "Particle Size \& Zeta Potential Analyzer" (Particulate Systems a division of Micromeritics, 4356 Communications Drive, Norcross GA, 30093, USA).

\section{Stability assay of EB + NFs against UV radiation}

The stability of the EB + NFs against ultraviolet (UV) radiation was tested by exposing the samples to a $36 \mathrm{~W}$ germicidal lamp $(254 \mathrm{~nm})$ at a distance of $20 \mathrm{~cm}$ at room temperature. The samples were withdrawn every $12 \mathrm{~h}$ in the $72 \mathrm{~h}$ for analysis and the changes of the EB content were analyzed by HPLC. The methanol solution of the EB (a.i.) was used as the control sample at the same time. ${ }^{11}$

\section{Release study}

The release experiments were carried out according to the method of Guo et al. ${ }^{11} 0.1 \mathrm{~g}$ from different EB + NFs were weighed and suspended in $100 \mathrm{~mL}$ of the methanol-water mixture $(30: 70, \mathrm{v} / \mathrm{v})$. This methanol-water mixture was used as a release medium in order to dissolve the EB. $5 \mathrm{~mL}$ suspension of the EB + NFs was introduced into a dialysis bags and stirred at a speed of $100 \mathrm{rpm}$ at room temperature, then the released EB from the dialysis bags was monitored up to $72 \mathrm{~h}$. The released solution was collected at different intervals after $(2,4,6,12,24$, $36,48,60$ and $72 \mathrm{~h}$ ), and centrifuged at speed $10000 \mathrm{rpm}$ for $15 \mathrm{~min}$ at room temperature. The concentration in the supernatant was determined using HPLC and the cumulative release rate of the EB from the EB + NFs was calculated to evaluate the sustained release properties. Data of the gradual release curve from $\mathrm{EB}+\mathrm{NFs}$ release experiments were fitted to the following equation. ${ }^{18,19}$

$$
\frac{M_{t}}{M_{a \infty}}=k t^{n}
$$

$M_{t}$ is the amount of EB released at time $t, M_{a \infty}$ is the total amount of EB in EB + NFs, $k$ is a release constant and $n$ is a diffusional exponent.

\section{Insect culture}

The initial population of $P$. xylostella was collected from a cruciferous vegetable field in the eastern suburbs of Hangzhou $\left(30^{\circ} 14^{\prime} \mathrm{N}, 120^{\circ} 15^{\prime} \mathrm{E}\right)$, Zhejiang Province, China, in September 2014. P. xylostella was reared at $25 \pm 2{ }^{\circ} \mathrm{C}$ with $70 \% \pm$ $10 \%$ relative humidity $(\mathrm{RH})$ and a light-dark cycle of $16: 8 \mathrm{~h}$. The larvae were reared on cabbage leaves (Brassica oleracea var. capitata (L.) (Capparales: Brassicaceae) cv. Jingfeng No. 1), and adults were fed with $10 \%$ sucrose solution. ${ }^{20}$

\section{Bioassay experiments}

The bioassay for the efficacy of EB + NFs against the early third instar larvae of $P$. xylostella was performed in plastic containers $(14.2 \times 7.2 \times 5.2 \mathrm{~cm})$ using leaf dipping method and carried out at $25 \pm 2{ }^{\circ} \mathrm{C}$ with $70 \% \pm 10 \% \mathrm{RH}$ and a light-dark cycle of $16: 8$ h. Dilutions of EB + NFs and EB were prepared using ultrapure water. The concentrations were prepared based on the mortality rang falling between $20 \%$ and $80 \%,{ }^{21}$ and the total volume was $100 \mathrm{~mL}$ for each concentration. Leaf discs $(2.5 \mathrm{~cm}$ in diameter) of cabbage leaves were dipped for $30 \mathrm{~s}$ in the test solution with gentle agitation. The leaf discs dipped in ultrapure water were served as control. Thirty min later, the surface of leaf discs was air dried, one dipped leaf disc with 30 early third instar larvae was placed in a perforated plastic container. The larvae were allowed to feed on the treated leaf disc for $24 \mathrm{~h}$, and then fed them with clean leaf disc until the end of the experiment. Insect mortality was recorded at 24,48 , and $72 \mathrm{~h}$ after the larvae were exposed to EB + NFs or EB alone. Three replicates for each concentration were performed, and 30 larvae were utilized for each replicate.

\section{Statistical analysis}

The mortality was analyzed via a two-way analysis of variance (ANOVA) with IBM SPSS Statistics 19 software. All percentage data were transformed using arcsine square root before ANOVA to standardize means and normalize variances and were transformed back to percentage for presentation. Mean values were separated through the least significant difference (LSD) test $(P<0.05)$ when significant differences among several mean values were detected by ANOVA. The $\mathrm{LC}_{50}$ values were calculated using the statistical method of LdP line program software (http:/embakr.tripod.com/ldpline/ldpline.htm), which was devoted to the calculation of probit analysis based on Finney's method. ${ }^{22,23}$

\section{Results and discussion}

\section{Encapsulation efficiency of EB + NFs}

The standard curve of EB showed a good linear relationship between the concentrations that ranged from 5 to $100 \mathrm{mg} \mathrm{L}^{-1}$

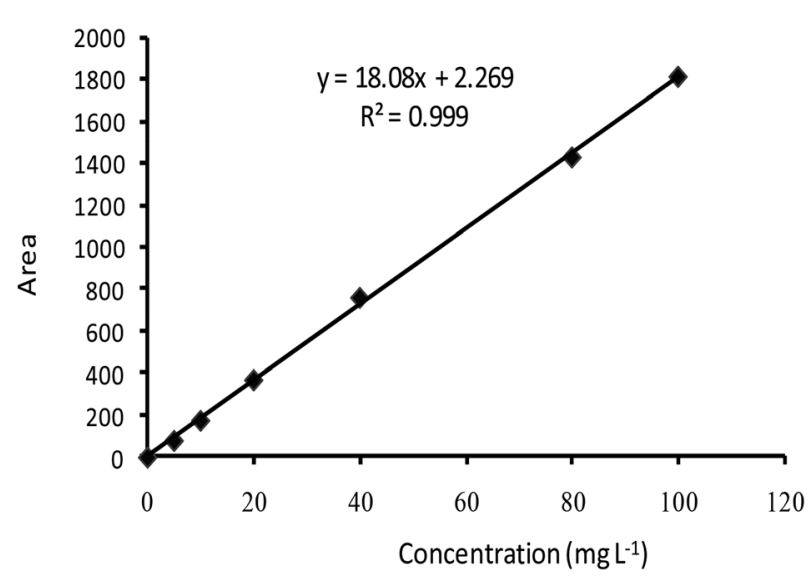

Fig. 1 Standard curve of emamectin benzoate. 
Table 1 Characterization of EB + NFs

\begin{tabular}{lllll}
\hline Formulation & Absolute recovery AR (\%) & Entrapment efficiency EE $(\%)$ & Particle size (nm) \pm SD & Zeta potential (mV) \pm SD \\
\hline EB + PNC & 72.51 & 92.84 & $219.93 \pm 3.89$ & $-26.43 \pm 2.90$ \\
EB + SNPs & 94.55 & 87.45 & $142.77 \pm 3.43$ & $-41.00 \pm 1.31$ \\
EB + MCM-48 & 74.29 & 71.19 & $119.73 \pm 20.28$ & $-36.50 \pm 0.56$
\end{tabular}
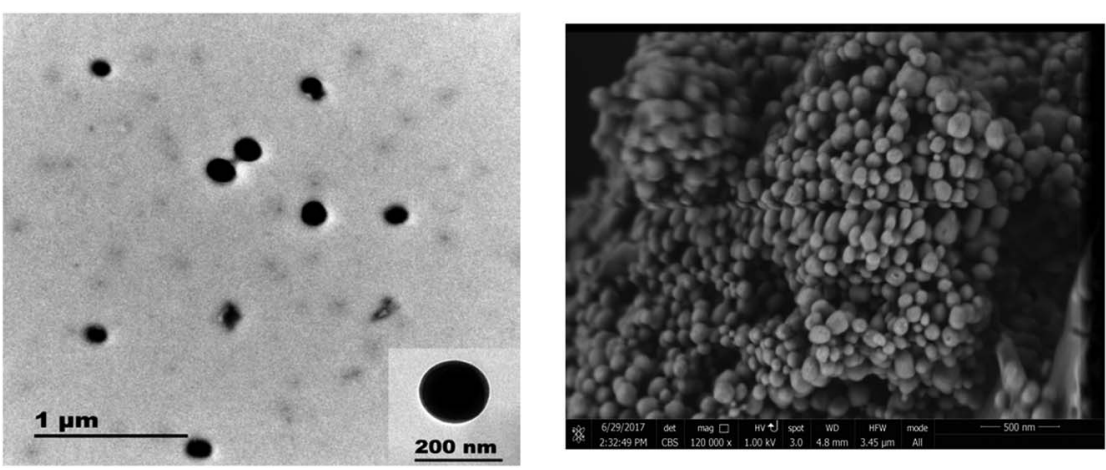

Fig. 2 TEM (left) and SEM (right) image of EB + PNC.

with linear equation $y=18.08 x-2.2698\left(R^{2}=0.9994\right)$ (Fig. 1). The AR\% was $72.51 \%, 94.55 \%$ and $74.29 \%$, and EE\% of EB + NFs was $92.84 \%, 87.45 \%$ and $71.19 \%$ from EB + PNC, EB + SNPs and EB + MCM-48, respectively (Table 1).

The ethyl cellulose, SNPs and MCM-48 were used to improve the EB formulations to avoid the disadvantage and the side effects of pesticides. According to the obtained EE\% results, the NFs preparation method is suitable for preparation of $\mathrm{EB}+\mathrm{NFs}$.

Ethyl cellulose that are used in preparation of $\mathrm{EB}+\mathrm{PNC}$ showed a higher encapsulation rate than other nanocapsule formulations, this may be due to its physical and chemical properties and its high ability to encapsulate the active ingredient in the nanocapsules. ${ }^{24,25}$

The EE\% of MCM-48 for EB reached to $71.19 \%$. The efficacy of MCM-48 for EB adsorption is due to its large surface area, porous structure and small particle size. The pore diameter also plays an important role in the loading process. The MCM-48 channels porous diameter is larger than the sectional diameter of EB molecules, allowing EB molecules to be entrapped into the porous structure of MCM-48 nanosilica. Furthermore, the type of MCM-48 nanosilica has a 3D cubic mesoporous structure with open networks and high surface area. ${ }^{14}$ This structure increase its adsorption capacity of the EB. Our results support several earlier studies. . $^{13,14,26,27}$ The amount of avermectin encapsulated in the MSNs reached $58.3 \% \mathrm{w} / \mathrm{w}$ by a simple immersion loading method, thus most of the adsorption of avermectin on the mesoporous nanosilica might be physical. ${ }^{26}$ The MSNs with a shell thickness of $\sim 15 \mathrm{~nm}$ and a pore diameter of $4-5 \mathrm{~nm}$ have an encapsulation capacity of $625 \mathrm{~g} \mathrm{~kg}^{-1}$ for avermectin using a supercritical fluid loading method. ${ }^{27}$ Similarly, MCM-48 nanospheres can be utilized as an effective delivery carrier owing to their high surface area and unique 3D open pore structure. ${ }^{14}$ In addition, the MSNs showed excellent pesticide loading capacity and delivery performance in controlled release, anti-photolysis, and water dispersity of abamectin. ${ }^{13}$

Pesticide loading can be achieved by different methods on the nanoparticles carriers such as extrusion, spray dry and freeze dry. ${ }^{28} \mathrm{~A}$ successful colloidal delivery system should have a high loading capacity. In this study, the EE of EB + SNPs reached to $87.45 \%$, the high loading capacity maybe due to the competition between its solubility in the water and its adsorption on to the SNPs surface. Also because the EB is poorly soluble in aqueous media and its ability to be adsorbed on the SNPs is higher than its solubility in the water. In addition, silica gel is a widely employed compound in the column

Table 2 Analysis of variance of the main parameters and their interactions

\begin{tabular}{|c|c|c|c|c|c|c|c|c|}
\hline \multirow[b]{2}{*}{ Source } & \multicolumn{2}{|l|}{ EB } & \multicolumn{2}{|c|}{$\mathrm{EB}+\mathrm{PNC}$} & \multicolumn{2}{|c|}{$\mathrm{EB}+\mathrm{SNPs}$} & \multicolumn{2}{|c|}{$\mathrm{EB}+\mathrm{MCM}-48$} \\
\hline & $F$ value & $P$ value & $F$ value & $P$ value & $F$ value & $P$ value & $F$ value & $P$ value \\
\hline Conc. & 392.98 & 0.0001 & 580.83 & 0.0001 & 1776.89 & 0.0001 & 221.82 & 0.0001 \\
\hline Time* conc. & 21.12 & 0.0001 & 32.36 & 0.0001 & 20.07 & 0.0001 & 4.8 & 0.0001 \\
\hline
\end{tabular}


chromatography as a stationary phase and as adsorbents in the environmental studies because it has high adsorption potential of organic and inorganic compounds. ${ }^{29}$

\section{Characterization of the EB + NFs}

Analyses of morphology and sizes of EB + NFs. Regarding to $\mathrm{EB}+\mathrm{PNC}$, according to TEM micrographs, the EB has been successfully encapsulated in the nanocapsules of ethyl cellulose (Fig. 2) and the nanocapsules were of spherical in shape with an average size of $219.93 \pm 3.89 \mathrm{~nm}$ (Table 2). SEM micrographs also revealed that the EB + PNC had a homogeneous distribution of particles (Fig. 2). Regarding to EB + SNPs, TEM image showed that the SNPs had nanometric sizes (Fig. 3). The SNPs

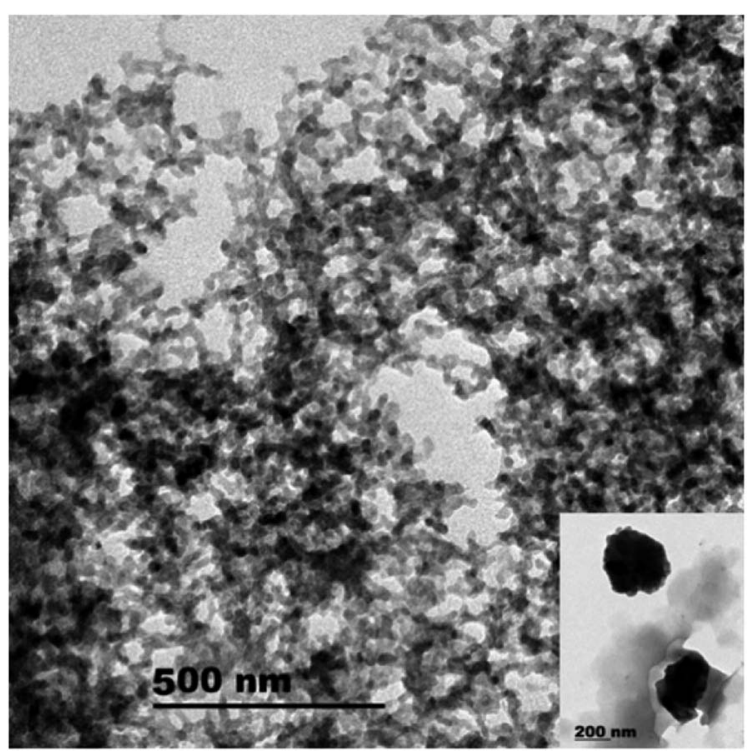

Fig. 3 TEM image of SNPS.

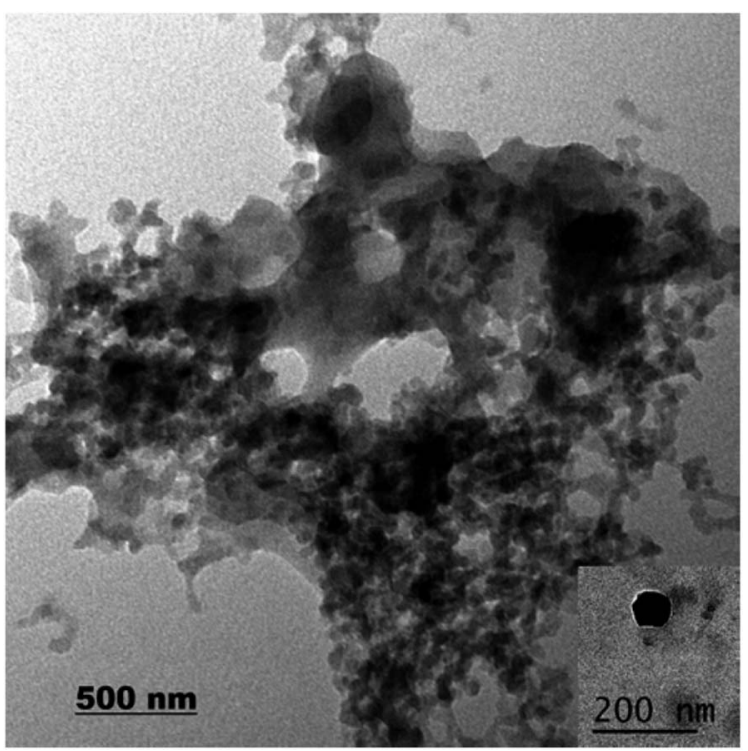

Fig. 4 TEM image of EB + SNPs had spherical shape with a small percentage of irregular surfaces, TEM image showed that the EB adsorbed by SNPs and created thin film around the SNPs (Fig. 4), and the average size was around $142.77 \pm 3.43 \mathrm{~nm}$ (Table 1 ). The TEM image of the MCM-48 is presented in Fig. 5. The TEM micrographs showed that core-shell-structured silica nanoparticles, with an average size of $119.73 \pm 20.28 \mathrm{~nm}$ (Table 1 ) and a shell thickness of $10 \sim$ $15 \mathrm{~nm}$. The EB was incorporated in pores of MSNs to obtain controlled release formulation. The pore size of MCM-48 wall is larger than the diameter of EB, allowing EB molecules to be entrapped into the MCM-48 (Fig. 6).

The well-known ethyl cellulose is one of the most constructive polymer, and is used widely to synthesize the microcapsules and nanocapsules for drugs and pesticides because of its

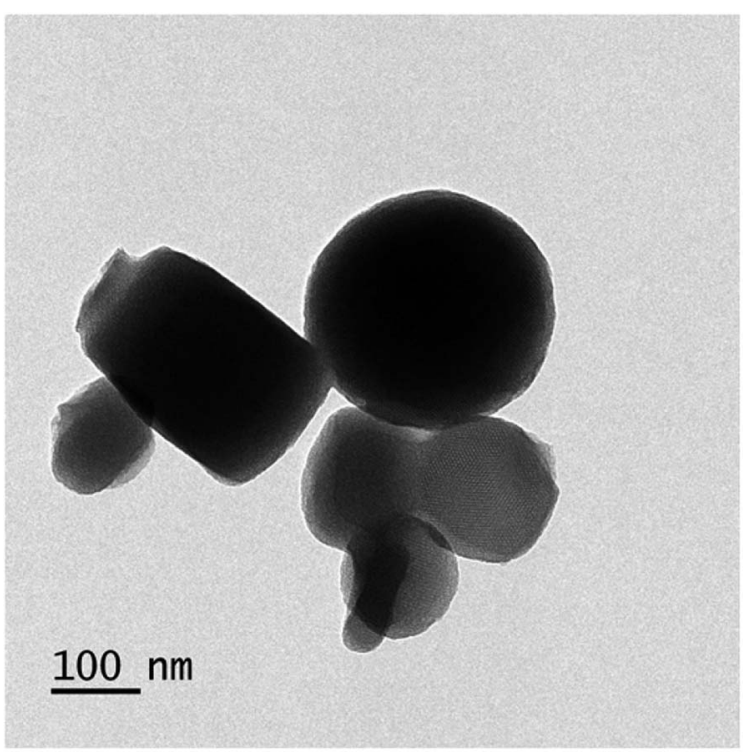

Fig. 5 TEM image of MCM-48.

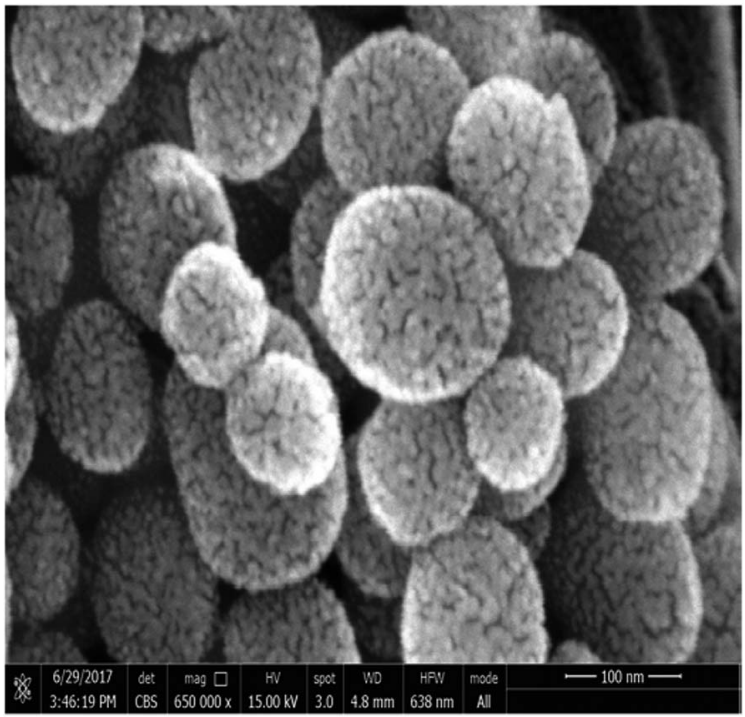

Fig. 6 SEM image of EB + MCM-48. 

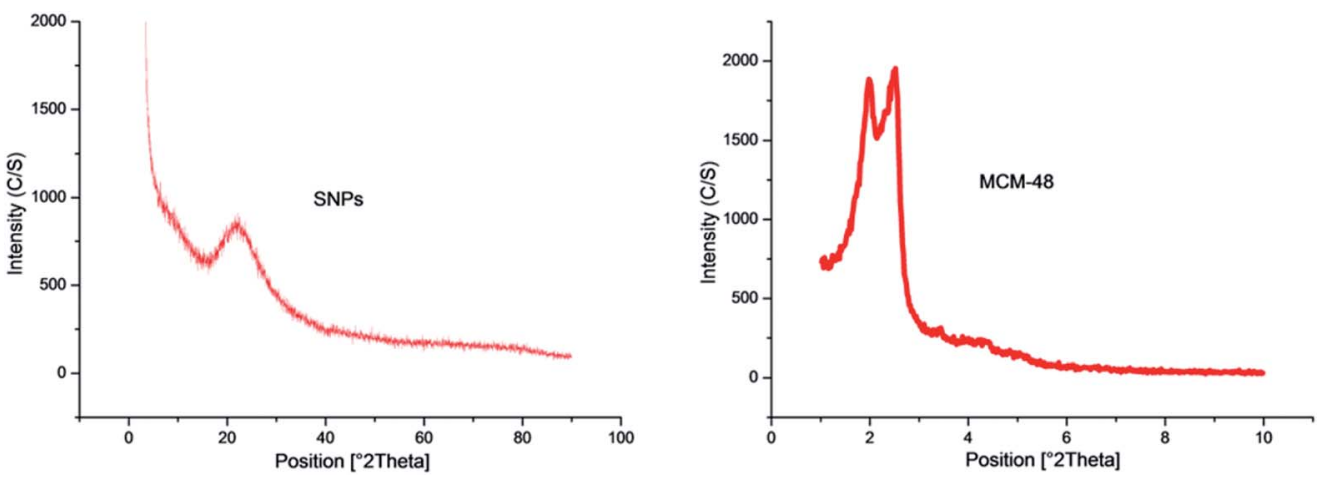

Fig. 7 XRD pattern of SNPs and MCM-48 nanosilica.

advantages as formulator, such as perfect film formability, higher physical-chemical stability, and minimum toxicity ${ }^{24,25,30,31}$ Regarding MCM-48, the MCM-48 has an average diameter of $119.73 \pm 20.28 \mathrm{~nm}$ with a spherical in morphology and a highly organized porous structure, and it has similar characteristic reported by Popat et al. ${ }^{14}$

The freeze-drying technique is used to enhance the stability of colloidal nanoparticles which also include nanocarriers for CRFs of pesticides. It is widely used for drying the unstable or heat-sensitive compounds at low temperatures without damaging their chemical structure. In this study, the SNPs colloidal solution mixed with EB (a.i.), then the aqueous phase was frozen and subjected to a low-pressure system. When the pressure is reduced drastically, the water sublimates (goes from solid to vapor state) and the EB a.i. leaves on SNPs.

Regarding to XRD analysis. It was used to investigate the structure of SNPs and MCM-48 nanoparticles. The XRD patterns of SNPs and MCM-48 were showed in (Fig. 7). The SNPs peak was observed at $21.218^{\circ} \theta$ using Bragg's law, that is, $\lambda=2 d \operatorname{Sin} \theta$, and the MCM-48 peak was observed at $2.6^{\circ} \theta$. These results showed a broad peak for an amorphous nanosilica core region. The XRD pattern of SNPs was compatible with earlier studies

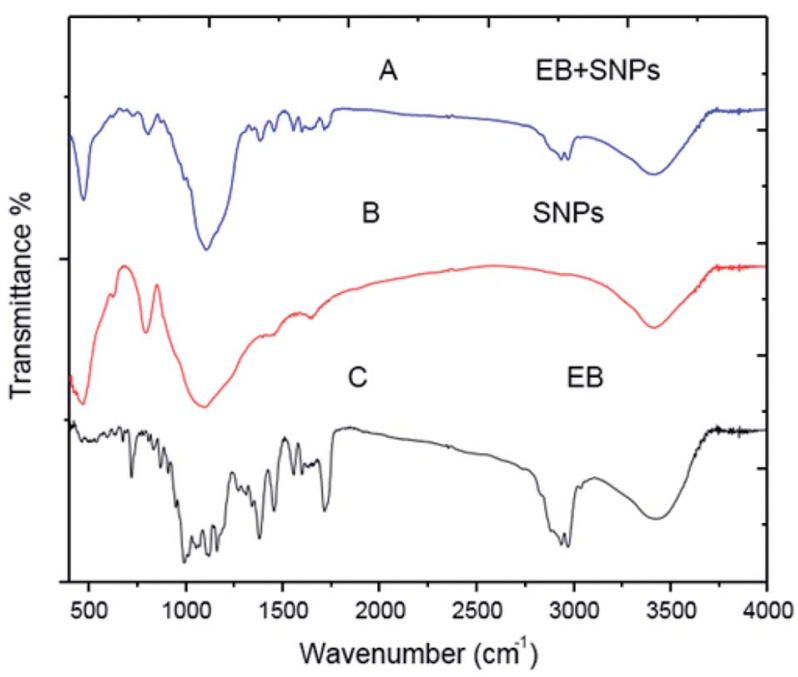

Fig. 8 FTIR spectrum of SNPs and EB + SNPs. which reported that the peak of SNPs was approximately at $20^{\circ}$ $\theta{ }^{32,33}$ Regarding to MCM-48 nanosilica, the obtained results established that the synthesized compound is MCM-48 nanosilica. This result was in accordance with Choi et al., ${ }^{34}$ they observed a sharp first Bragg peak indexed as (211) at $2^{\circ} \theta=2.51^{\circ}$ $\theta$ and the second peak (220) at $2.89^{\circ} \theta$ for the cubic Ia3d mesostructure (MCM-48). It is also compatible with previous studies which discussed the chemical and physical characterization for the MCM-48 nanosilica. ${ }^{35-37}$

FT-IR analysis. The infrared spectra of EB blank, SNPs, MCM-48, EB + SNPs and EB + MCM-48 samples showed in Fig. 8 and Fig. 9. Regarding to SNPs, the absorption band at $3419 \mathrm{~cm}^{-1}$ (Fig. 8 A) showed that only a small amount of water is present in the samples. The very broad strong peak at $1097 \mathrm{~cm}^{-1}$ can be ascribed to composite of $\mathrm{Si}-\mathrm{O}$ stretching of nanosilica (Fig. 8 B). For the free EB, the bands at 2967 and $2982 \mathrm{~cm}^{-1}$ are attributed to $\mathrm{C}-\mathrm{H}$ stretching vibrations of an aromatic ring corresponding to the benzoate fraction or conjugated olefins, $1716 \mathrm{~cm}^{-1}$ bending vibration of $(\mathrm{C}=\mathrm{O}$ stretching vibrations of an acrylics ester), 1634, 1599 and $1557 \mathrm{~cm}^{-1}$ are ascribed to $(\mathrm{C}=\mathrm{C}$ stretching vibrations of an aromatic ring or conjugated olefins), 1455 and $1379 \mathrm{~cm}^{-1}$ are 
identified as skeleton vibration of $(\mathrm{C}-\mathrm{H}$ deformation in $\mathrm{CH} 3$ groups), 1160, 1118 and $1058 \mathrm{~cm}^{-1}$ are attributed to $(\mathrm{C}-\mathrm{O}$ stretching vibrations, $\mathrm{O}-\mathrm{H}$ and $\mathrm{C}-\mathrm{O}-\mathrm{C}$ flexion), $991 \mathrm{~cm}^{-1}$ bending vibration of $(\mathrm{C}-\mathrm{H}$ flexion of trans $\mathrm{C}=\mathrm{C}$ bonding $)$ and 947-568 $\mathrm{cm}^{-1}(\mathrm{C}-\mathrm{H}$ flexion outside the plane in an aromatic ring or $\mathrm{C}=\mathrm{C}$ cis bond) (Fig. $8 \mathrm{C}$ ). For the $\mathrm{EB}+\mathrm{SNPs}$, the spectrum retained most of the major peaks of SNPs and EB, and no noticeable new peaks were observed in EB + SNPs (Fig. 8A). The FT IR of free EB blank, MCM-48 and EB + MCM-48 showed in Fig9. The obtained results for blank MCM-48 were similar with SNPs spectrum that mentioned above, and the FT IR of EB, was mentioned above (Fig. 9C). The very broad strong peak at 1090 $\mathrm{cm}^{1}$ can be ascribed to composite of Si-O stretching of nanosilica (Fig. 9B) and no noticeable new peaks were observed in EB + MCM-48 (Fig. 9A).

The FT-IR analysis was employed to examine possible interactions between the EB and the nanosilica carriers. The FTIR results confirmed that the EB + NFs spectrums retained most of the major peaks of pure carriers (SNPs and MCM-48) and EB, and did not show noticeable new peaks, indicating that the adsorption of EB in the NPs carriers is probably physical adsorption. Therefore, the properties of EB have not been changed after their loading on the NPs carriers. These results were convenient with the absorption behavior of avermectinPHSN as reported by Wen et $a .^{\mathbf{2 6}}$

Zeta potential. The zeta potential degree describes the electrostatic repulsion degree between adjacent, similarly charged particles in dispersion. The colloidal solution with $\mathrm{ZP}$ of $>$ $+30 \mathrm{mV}$ or $<-30 \mathrm{mV}$ is considered to be very stable. The zeta potential values for all EB + NFs were fallen in the range of -26 to $-41 \mathrm{mV}$ (Table 1). Zeta potential is an excellent technique for describing the properties of the nanoparticle surface and predicting the long term existence of the nanoparticle. In the low zeta potential, attractive forces may overcome this repulsion and the dispersion may split and flocculate. So, the colloidal system with high zeta potential are electrically stabilized while the colloidal system with low zeta potentials are electrically unstable. ${ }^{38,39}$ Especially, the NPs with zeta potential of $>+30 \mathrm{mV}$

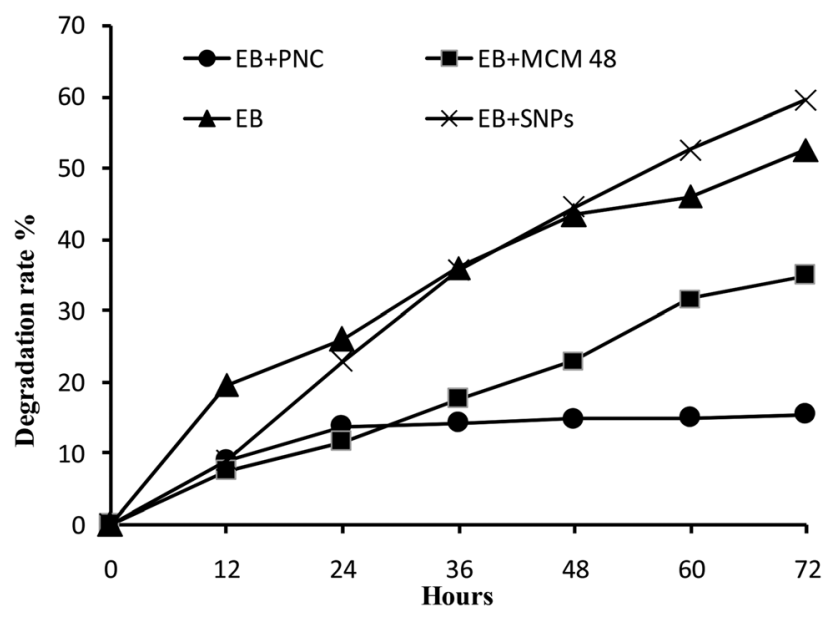

Fig. 10 Stability of the resulting EB + NFs and the active ingredient against UV radiation. or $<-30 \mathrm{mV}$ is considered to be very stable in the dispersion medium. ${ }^{\mathbf{1 , 4 0}}$ In our study, the zeta potential values for all EB + NFs were more than $-26 \mathrm{mV}$, suggesting that the NPs formulations were stable in the dispersion medium.

UV stability. The photo-stability of EB + NFs and the EB alone showed in Fig. 10. The degradation rates were 15.35, 34.94, 52.58 and $59.50 \%$ for EB + PNC, EB + MCM-48, EB and EB + SNPs after exposure of $72 \mathrm{~h}$, respectively. These results showed that the EB + PNC was more stable than all other samples, and no significant difference was detected between EB and EB + SNPs. The UV radiation results showed that the EB could be protected through the EB + PNC and EB + MCM-48, nanosilica wall can significantly prevent the photolysis of EB and increase the EB stability. While the EB + SNPs did not protect EB from photolysis because EB was only adsorbed on the surface of SNPs.

Protecting the a.i. of a formulation under field conditions is necessary when the local environment adversely affects the stability of the pesticides. So, the encapsulation is necessary to overcome the stability problems of a.i. and also to improve the solubility of this pesticide in water. ${ }^{28}$

With regard to $\mathrm{EB}+\mathrm{PNC}$, the ethyl cellulose enhanced the photostability of EB + PNC, the stability against UV radiation maybe due to the physical and chemical characteristics of ethyl cellulose. The ethyl cellulose has been widely used for microencapsulation due to its versatile properties such as melting point range $240-255{ }^{\circ} \mathrm{C}$, specific density range $1.07-1.18$ with 135-155 ${ }^{\circ} \mathrm{C}$ heat distortion point and $330-360{ }^{\circ} \mathrm{C}$ fire point, stability against light, heat, wetness and chemicals, and ability to absorb pressure. ${ }^{41}$ Because, the polymeric chain forms a stronger film isolating the a.i. from the external environment, it can protect the a.i. from the degradation by UV. The previous studies confirmed that the polymeric nanocapsules could improve the persistence of a.i. against UV radiation such as natural products, ${ }^{1}$ acetamiprid microcapsules ${ }^{4}$ and the EB slow release microspheres. ${ }^{42}$ Consequently, the polymeric nanocapsule is able to protect the a.i. from the rapid degradation or may increase the efficiency of pest control for a long duration. Furthermore, it can be able to lower the dosage of pesticides and exposure to human.

The EB + MCM-48 reduced significantly the degradation rate of EB. This is probably due to the EB was entrapped into the pores of MCM-48. These results were consistent with the previous study by Guo et al. ${ }^{\mathbf{1 1}}$ they found that the EB was sensitive to UV radiation and the samples were degraded completely within $48 \mathrm{~h}$, while the decomposition rate of the EB wrapped in microcapsules was less than $25 \%$ after $72 \mathrm{~h}$ of UV exposure. On the other hand, the MSNs highly improved the photostability of avermectin by entrapping it into the hollow core of the nanoparticle carriers. ${ }^{12,26}$ The MSNs not only can protect the active ingredient from UV radiation but also can enhance the chemical solubility and its dispersity in the water. ${ }^{13}$ Improvement of the insecticides stability can reduce the concentration of insecticides in commercial spray applications, without lowering the efficiency. These kinds of the formulations $(\mathrm{EB}+\mathrm{PNC}$ and $\mathrm{EB}+\mathrm{MCM}-48)$ are convenient for application in the early stage of plant life, which requires stable pesticides 


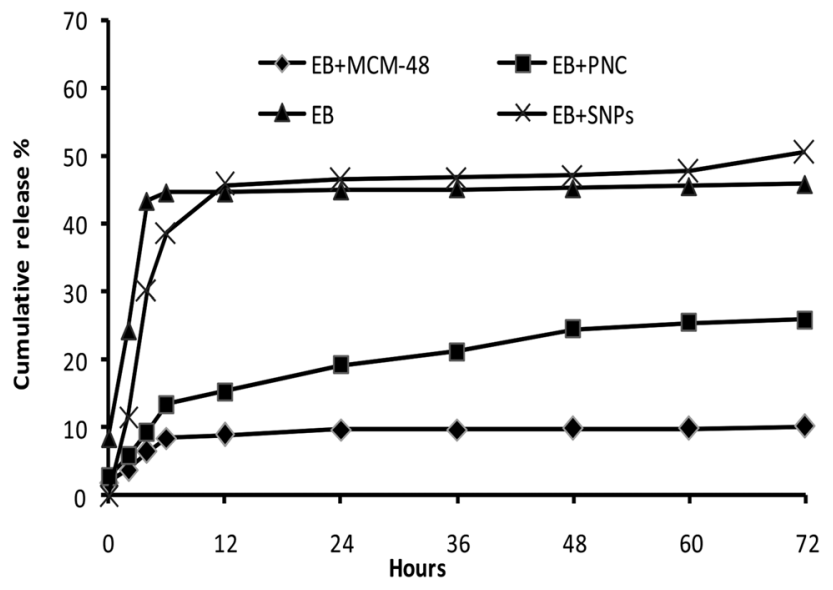

Fig. 11 The release behaviors of the EB and EB + NFs.

under various environmental conditions to protect the plant for long period. Consequently, they may reduce economic cost by decreasing the number of applications. Moreover, insecticide nanodelivery systems were proposed to increase the spatial distribution on leaf surfaces of crops due to the nanosize and thereby enhance the effectiveness of pesticide applications. Besides, pesticide nanodelivery systems also have better penetration ability through the cuticle, and allow slow and controlled release of active ingredients on the target. ${ }^{13}$

The EB and EB + SNPs showed no significant difference in the stability against UV radiation. The SNPs do not has the ability to protect the a.i., this may be due to that the active ingredient in EB + SNPs were adsorbed on the surface of the SNPs, and exposed directly to UV radiation.

The release behaviors of EB + NFs. The release profiles of EB + NFs showed in the Fig. 11. The releases of EB in all samples were relatively fast at initial stage and then progressively slow with increasing time. The cumulative release rates were 10.11, 25.84, 45.77 and $50.47 \%$ after $72 \mathrm{~h}$ for EB + MCM-48, EB + PNC, EB and EB + SNPs, respectively.

CRFs play a critical role to reduce the environmental problems associated with the application of pesticides. The colloidal delivery system has great potential to improve the pesticide CRFs and remarkably reduce effective dosage by maintaining an effective concentration in the target for longer periods of time. ${ }^{13}$ In this study, the EB + MCM-48 showed two stages in release behavior. The release in the first few hours was so fast, then, the release rate became much slower in the later hours. In the first stage, it may be due to the dissolvent of the EB adsorbed on the external surface of MCM-48, while in the second stage, it may be due to the hindrance of the porous structure of MCM- 48 . These results showed good agreement with the release behaviors of avermectin porous hollow silica nanoparticles (avermectinPHSNs), abamectin-PHSNs and imidacloprid-mesoporous silica nanoparticles formulations. ${ }^{13,14,26}$

The EB + PNC release behavior was similar to that of EB + MCM-48 release. The slow release profile may be due to the stronger mechanical property of the nanocapsules that prevent the excessive release of EB from the EB + PNC. Moreover, the

Table 3 Mortality (mean \pm SD) of the third instar larvae of $P$. xylostella after EB + NFs exposure via leaf dipping ${ }^{a}$

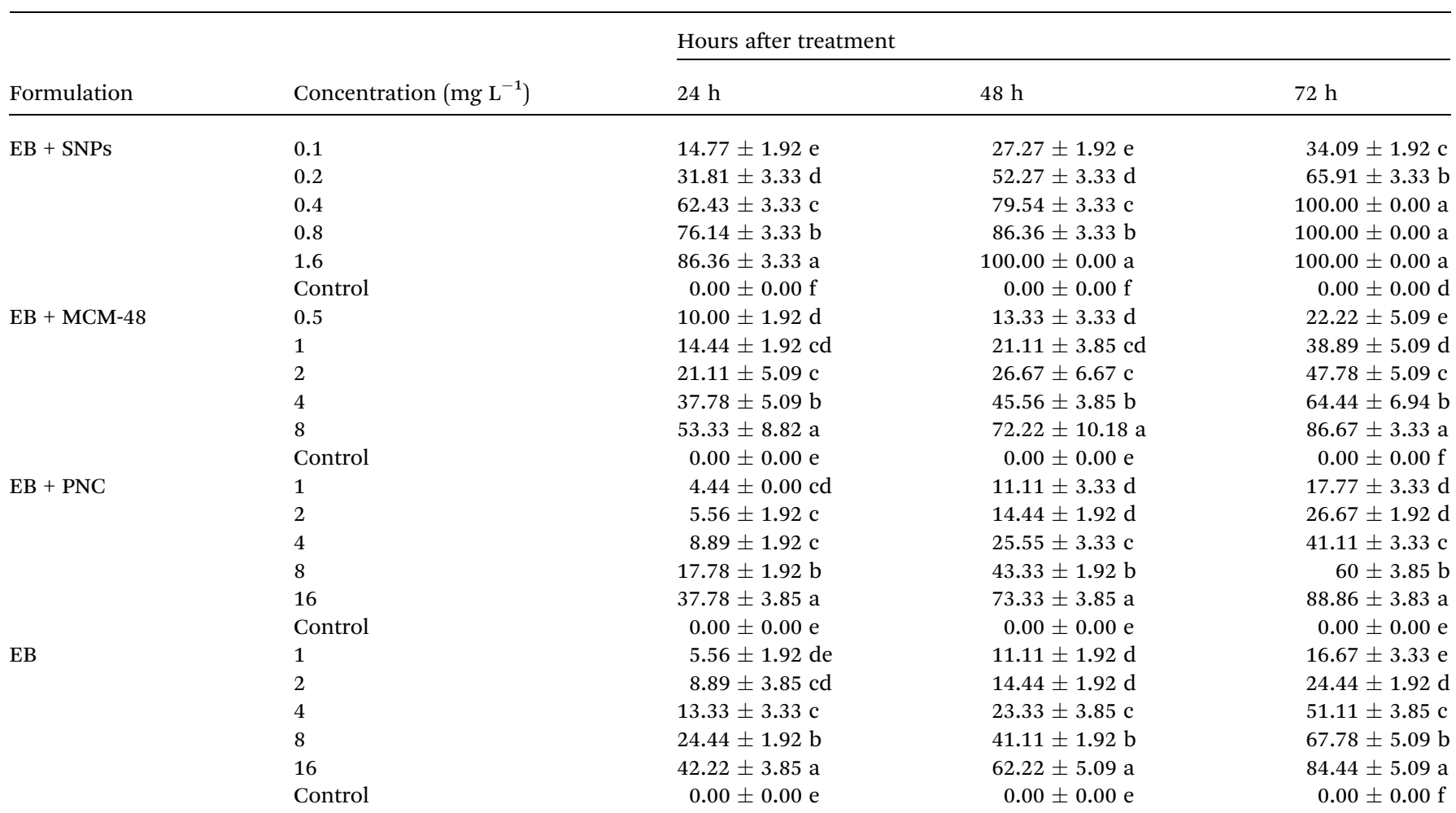

\footnotetext{
${ }^{a}$ Different letter in column under same formulation followed after mean $( \pm \mathrm{SD})$ indicate significant difference at $P=0.05$ level.
} 
Table $4 \quad L_{50-90}$ values of EB + NFs against the third instar larvae of $P$. xylostella ${ }^{a}$

\begin{tabular}{|c|c|c|c|c|c|}
\hline Formulation & Time (h) & $\mathrm{LC}_{50}\left(\mathrm{mg} \mathrm{L}^{-1}\right)(\mathrm{LCL}-\mathrm{UCL})$ & $\mathrm{LC}_{90}\left(\mathrm{mg} \mathrm{L}^{-1}\right)(\mathrm{LCL}-\mathrm{UCL})$ & Slope \pm SE & $x^{2}$ \\
\hline \multirow[t]{2}{*}{$\mathrm{EB}+\mathrm{SNPs}$} & 24 & $0.32(0.27-0.37)$ & $1.67(1.26-2.47)$ & $1.78 \pm 0.16$ & 2.66 \\
\hline & 48 & $0.18(0.15-0.21)$ & $0.75(0.60-0.99)$ & $2.07 \pm 0.19$ & 3.47 \\
\hline & 48 & $4.03(3.23-5.34)$ & 33.81 (19.96-76.32) & $1.38 \pm 0.16$ & 5.05 \\
\hline & 72 & $1.80(1.46-2.21)$ & $14.27(9.61-25.64)$ & $1.42 \pm 0.15$ & 3.32 \\
\hline $\mathrm{EB}+\mathrm{PNC}$ & 24 & $34.79(21.51-82.29)$ & 359.51 (131.92-2392.08) & $1.26 \pm 0.22$ & 3.23 \\
\hline \multirow[t]{3}{*}{ EB } & 24 & $24.83(17.41-56.21)$ & $311.32(119.88-1783.47)$ & $1.20 \pm 0.18$ & 1.32 \\
\hline & 48 & $11.06(8.56-15.75)$ & $99.64(53.95-265.99)$ & $1.34 \pm 0.16$ & 2.70 \\
\hline & 72 & $4.22(3.55-5.04)$ & $24.14(17.46-38.08)$ & $1.69 \pm 0.16$ & 1.70 \\
\hline
\end{tabular}

denser ethyl cellulose polymer chains and its physicochemical properties led to the reduction in release. ${ }^{24,25}$ The release profiles of EB both in EB + SNPs and in the control (EB alone) showed no significant difference. Both showed a faster release at first $12 \mathrm{~h}$, this may be due to the fast dissolution of EB on the surface of SNPs.

Bioassay. All main effects and their associated interactions were significant at $P<0.0001$ level (Table 2). In all treatments, mortality percentage increased with the increase in concentrations and with the passage of time (Table 3). The efficacy of EB + $\mathrm{NFs}$ was compared on the $\mathrm{LC}_{50}$ values. Results showed that the EB + SNPs is more effective than other treatments, because it showed $100 \%$ mortality after $48 \mathrm{~h}$ but other treatments $\mathrm{EB}+$ MCM-48, EB and EB + PNC showed 86.67\%, 88.86\% and $84.44 \%$ mortality after $72 \mathrm{~h}$ respectively (Table 3 ). The lowest $\mathrm{LC}_{50}$ was recorded when larvae were exposed to EB + SNPs, followed by EB + MCM-48 which are much lower than EB + PNC and EB. However, no significant difference was observed between the EB + PNC and the EB. The $\mathrm{LC}_{50}$ value of $\mathrm{EB}+\mathrm{SNPs}$ was $0.18 \mathrm{mg} \mathrm{L}^{-1}$ after $48 \mathrm{~h}$, while the $\mathrm{LC}_{50}$ values for other treatments were 1.8, 4.22 and $4.52 \mathrm{mg} \mathrm{L}^{-1}$ of EB + MCM-48, EB and EB + PNC after $72 \mathrm{~h}$, respectively (Table 4).

The improved efficacy of EB + SNPs and EB + MCM-48 may be due to the smaller particle size, higher surface area and their high mobility ratio, which eventually lead to increasing penetration of NPs formulations in the larval body than the active ingredient alone. The surface-functionalized silica nanoparticles can deliver DNA and drugs into animal cells and tissues, ${ }^{43}$ because nanoparticles drug carriers have the potential to cross physiological barriers and access different tissues. ${ }^{44}$ The insecticidal activity of pyridalyl nanosuspension was more effective than the commercial formulation and was 2.26 and 6.25 times more effective against $H$. armigera as stomach poison than the technical product and commercial formulation respectively. ${ }^{45}$ They thought that the increased toxicity of nano sized formulation on larvae is probably due to increasing penetration of pyridalyl in the larval body.

Regarding to the effectiveness of EB + PNC and EB, a little difference was noticed between their effectiveness. At the equal concentration, EB showed more effective than that of the EB +
PNC in the first day, whereas EB + PNC was more effective than $\mathrm{EB}$ alone during the second day. These results may be due to the efficacy of EB + PNC is dependent on the controlled release of the a.i. from the nanocapsules. These results are consistence with the findings of Guo et al. ${ }^{11}$ who reported that when treated M. persicae at the same concentration, EB $1 \%$ EC is more effective than that of the microcapsules at first day after treatment. Similar results were also reported by Zhang et al., where the effectiveness of phoxim microcapsules increased with the passage of times. ${ }^{46}$ The similar results could be due to that the insecticides were loaded on the similar carrier with similar physicochemical properties.

\section{Conclusions}

In conclusion, according to our study, we can suggest that the colloidal delivery systems such as SNPs, MCM-48 and PNC could act as a controlled release carrier and can maintain chemical stability of EB. They may overcome environmental sensitivity and poor water solubility, and increase the efficacy of insecticides. These advantages could eventually lead to minimize the dosage of pesticides needed, reducing the number of applications required in comparison to conventional formulations and decreasing pesticides release in the environment. However, there are necessaries to study the safety issues regarding pesticide nanoformulations on the beneficial insects and human health.

\section{Conflicts of interest}

No potential conflict of interest was reported by the authors.

\section{Acknowledgements}

We acknowledge the National Basic Research Program of China (973 Program) [grant number 2009CB119005], [grant number 2006CB102005]; the National 948 Program [grant number 2011G4]; the National Department Benefit Research Foundation [grant number nyhyzx20110321] for their generous financial support to this research. 


\section{References}

1 M. R. Forim, E. S. Costa, M. F. G. F. da Silva, J. B. Fernandes, J. M. Mondego and A. L. Boiça Junior, J. Agric. Food Chem., 2013, 61, 9131-9139.

2 W. Krämer and U. Schirmer, in Modern Crop Protection Compounds, Wiley, Weinheim, 2007, pp. 1069-1086.

3 R. Jansson, R. Brown, B. Cartwright, D. Cox, D. Dunbar, R. Dybas, C. Eckel, J. Lasota, P. Mookerjee and J. Norton, Proceedings of the 3rd International Workshop on Management of Diamondback Moth and Other Crucifer Pests, MARDI, Kuala Lumpur, Malaysia, 1997.

4 Y. Cao, L. Huang, J. Chen, J. Liang, S. Long and Y. Lu, Int. J. Pharm., 2005, 298, 108-116.

5 A. Akelah, Mater. Sci. Eng. C, 1996, 4, 83-98.

6 A. Akelah, in Functionalized Polymeric Materials in Agriculture and the Food Industry, Springer, London, 2013, pp. 133-192.

7 C. Charcosset, A. El-Harati and H. Fessi, J. Controlled Release, 2005, 108, 112-120.

8 S. R. Choudhury, S. Pradhan and A. Goswami, Nanosci. Methods, 2012, 1, 9-15.

9 S. Pradhan, I. Roy, G. Lodh, P. Patra, S. R. Choudhury, A. Samanta and A. Goswami, J. Environ. Sci. Health, Part B, 2013, 48, 559-569.

10 F.-L. Yang, X.-G. Li, F. Zhu and C.-L. Lei, J. Agric. Food Chem., 2009, 57, 10156-10162.

11 M. Guo, W. Zhang, G. Ding, D. Guo, J. Zhu, B. Wang, D. Punyapitak and Y. Cao, RSC Adv., 2015, 5, 93170-93179.

12 Z.-Z. Li, J.-F. Chen, F. Liu, A.-Q. Liu, Q. Wang, H.-Y. Sun and L.-X. Wen, Pest Manage. Sci., 2007, 63, 241-246.

13 Y. Wang, H. Cui, C. Sun, X. Zhao and B. Cui, Nanoscale Res. Lett., 2014, 9, 655.

14 A. Popat, J. Liu, Q. Hu, M. Kennedy, B. Peters, G. Q. M. Lu and S. Z. Qiao, Nanoscale, 2012, 4, 970-975.

15 W. Meyer, P. Gurman, L. Stelinski and N. Elman, Green Chem., 2015, 17, 4173-4177.

16 S. Musić, N. Filipović-Vinceković and L. Sekovanić, Braz. J. Chem. Eng., 2011, 28, 89-94.

17 T.-W. Kim, P.-W. Chung and V. S.-Y. Lin, Chem. Mater., 2010, 22, 5093-5104.

18 Y. Wang, Z. Gao, F. Shen, Y. Li, S. Zhang, X. Ren and S. Hu, J. Agric. Food Chem., 2015, 63, 5196-5204.

19 R. W. Korsmeyer, R. Gurny, E. Doelker, P. Buri and N. A. Peppas, Int. J. Pharm., 1983, 15, 25-35.

20 X. Gu, S. Kumar, E. Kim and Y. Kim, J. Insect Physiol., 2015, 80, 81-87.

21 J. L. Robertson, K. C. Smith, N. Savin and R. J. Lavigne, J. Econ. Entomol., 1984, 77, 833-837.

22 W. Abbott, J. Econ. Entomol., 1925, 18, 265-267.

23 D. Finney, Probit Analysis, Cambridge University Press, London, 3rd edn, 1971.
24 M. Fernández-Pérez, M. Villafranca-Sánchez, F. FloresCéspedes and I. Daza-Fernández, Carbohydr. Polym., 2011, 83, 1672-1679.

25 B. S. Rao and K. R. Murthy, Int. J. Pharm., 2002, 231, 97-106.

26 L.-X. Wen, Z.-Z. Li, -H.-K. Zou, A.-Q. Liu and J.-F. Chen, Pest Manage. Sci., 2005, 61, 583-590.

27 A. H. Sayyed and D. J. Wright, Pest Manage. Sci., 2006, 62, 1045-1051.

28 B. Perlatti, J. B. Fernandes, M. F. G. F. da Silva, M. R. Forim and P. L. de Souza Bergo, Insecticides-Development of Safer and More Effective Technologies, ed. S. Trdan, Intech, Rijeka, 2013, vol. 20, pp. 523-550.

29 A. K. Kushwaha, N. Gupta and M. Chattopadhyaya, Arabian J. Chem., 2017, 10, S81-S89.

30 A. B. Lokhande, S. Mishra, R. D. Kulkarni and J. B. Naik, J. Pharma Res., 2013, 7, 414-420.

31 M. A. Latheef, O. Dailey and E. Franz, Pesticide Formulations and Application Systems, ed. B. N. Devisetty, D. G. Chasin and P. D. Berger, American Society for Testing and Materials, Philadelphia, 1993, vol. 13, pp. 300-312.

32 N. Debnath, S. Mitra, S. Das and A. Goswami, Powder Technol., 2012, 221, 252-256.

33 C. Vani and U. Brindhaa, Int. J. Pharm. Bio. Sci., 2013, 4, B1108-B1118.

34 E. Choi, J. Lu, F. Tamanoi and J. I. Zink, Z. Anorg. Allg. Chem., 2014, 640, 588-594.

35 I. Izquierdo-Barba, E. Sousa, J. C. Doadrio, A. L. Doadrio, J. P. Pariente, A. Martínez, F. Babonneau and M. ValletRegí, J. Sol-Gel Sci. Technol., 2009, 50, 421-429.

36 P. N. Trikalitis, N. Ding, C. Malliakas, S. J. Billinge and M. G. Kanatzidis, J. Am. Chem. Soc., 2004, 126, 15326-15327.

37 K. Schumacher, P. I. Ravikovitch, A. Du Chesne, A. V. Neimark and K. K. Unger, Langmuir, 2000, 16, 46484654 .

38 R. W. OBrien, Faraday Discuss. Chem. Soc., 1990, 90, 301-312. 39 D. Hanaor, M. Michelazzi, C. Leonelli and C. C. Sorrell, J. Eur. Ceram. Soc., 2012, 32, 235-244.

40 S. Bhattacharjee, J. Controlled Release, 2016, 235, 337-351.

41 G. Murtaza, Acta Pol. Pharm., 2012, 69, 11-22.

42 Y. Wang, A. Wang, C. Wang, B. Cui, C. Sun, X. Zhao, Z. Zeng, Y. Shen, F. Gao, G. Liu and H. Cui, Sci. Rep., 2017, 7, 12761. 43 F. Torney, B. G. Trewyn, V. S.-Y. Lin and K. Wang, Nat. Nanotechnol., 2007, 2, 295-300.

44 J. M. Rosenholm, C. Sahlgren and M. Lindén, Nanoscale, 2010, 2, 1870-1883.

45 P. Saini, M. Gopal, R. Kumar and C. Srivastava, J. Environ. Sci. Health, Part B, 2014, 49, 344-351.

46 D.-X. Zhang, B.-X. Li, X.-P. Zhang, Z.-Q. Zhang, W.-C. Wang and F. Liu, J. Agric. Food Chem., 2016, 64, 2841-2846. 